\title{
Regularity Results for a Quasilinear Free Boundary Problem
}

\author{
Samia Challal $^{a}$ and Abdeslem Lyaghfouri ${ }^{b}$ \\ ${ }^{a}$ Glendon College, York University \\ Department of Mathematics, Toronto, Ontario, Canada \\ ${ }^{b}$ American University of Ras Al Khaimah \\ Department of Mathematics and Natural Sciences, Ras Al Khaimah, UAE \\ E-mail(corresp.): abdeslem. lyaghfouri@aurak.ac.ae \\ E-mail: schallal@glendon.yorku.ca
}

Received July 4, 2019; revised March 7, 2020; accepted March 9, 2020

\begin{abstract}
In this paper we prove local interior and boundary Lipschitz continuity of the solutions of a quasilinear free boundary problem. We also show that the free boundary is the union of graphs of lower semi-continuous functions.
\end{abstract}

Keywords: A-Laplacian, free boundary, Lipschitz continuity.

AMS Subject Classification: 35R35; 35J60.

\section{Introduction}

We consider the following problem

$$
\left\{\begin{array}{l}
\text { Find }(u, \chi) \in W^{1, A}(\Omega) \times L^{\infty}(\Omega) \text { such that: } \\
i) \quad 0 \leq u \leq M, \quad 0 \leq \chi \leq 1, \quad u(1-\chi)=0 \text { a.e. in } \Omega \\
i i) \quad \Delta_{A} u=-\operatorname{div}(\chi \mathrm{H}(\mathrm{x})) \quad \text { in }\left(\mathrm{W}_{0}^{1, \mathrm{~A}}(\Omega)\right)^{\prime}
\end{array}\right.
$$

where $\Omega$ is an open bounded domain of $\mathbb{R}^{n}, n \geq 2, x=\left(x_{1}, \ldots, x_{n}\right), M$ is a positive constant, $A(t)=\int_{0}^{t} a(s) d s$, and $\Delta_{A}$ is the $A$-Laplacian

$$
\Delta_{A} u=\operatorname{div}\left(\frac{\mathrm{a}(|\nabla \mathrm{u}|)}{|\nabla \mathrm{u}|} \nabla \mathrm{u}\right)
$$

Copyright (C) 2020 The Author(s). Published by VGTU Press

This is an Open Access article distributed under the terms of the Creative Commons Attribution License (http://creativecommons.org/licenses/by/4.0/), which permits unrestricted use, distribution, and reproduction in any medium, provided the original author and source are credited. 
with $a$ a $C^{1}$ function from $[0, \infty)$ to $[0, \infty)$ such that $a(0)=0$ and satisfies for some positive constants $a_{0}, a_{1}$

$$
a_{0} \leq \frac{t a^{\prime}(t)}{a(t)} \leq a_{1} \quad \forall t>0 .
$$

The reader will find a variety of examples of such functions in [13]. As a consequence of (1.2), we have the following monotonicity inequality [10]

$$
\left(\frac{a(|\xi|)}{|\xi|} \xi-\frac{a(|\zeta|)}{|\zeta|} \zeta\right) \cdot(\xi-\zeta)>0 \quad \forall \xi, \zeta \in \mathbb{R}^{n} \backslash\{0\}, \quad \xi \neq \zeta .
$$

The operator $\Delta_{A}$ is a generalization of the $p$-Laplacian $\left(a(t)=t^{p-1}, p>1\right)$, which is itself a generalization of Laplace operator $(a(t)=t)$. If we further allow the function $a$ to depend on $x$ (see $[14,26]$ ), our results can be extended to include more operators like the so-called $p(x)$-Laplacian when $a(t, x)=$ $t^{p(x)-1}$, with $p(x)$ a continuous function such that $p_{+}>p(x)>p_{-}>1$ for all $x$ in $\Omega$, where $p_{-}$and $p_{+}$are two positive numbers. However, in order to avoid complicated calculations, we will restrict ourselves to the case where $a$ is independent of $x$.

$H=\left(H_{1}, \ldots, H_{n}\right)$ is a vector function that satisfies for a positive constant $\bar{h}$

$$
\begin{gathered}
|H|_{\infty} \leq \bar{h}, \\
|\operatorname{div}(\mathrm{H})|_{\infty} \leq \overline{\mathrm{h}} .
\end{gathered}
$$

For the definition of the Orlicz-Sobolev space $W^{1, A}(\Omega)$ and its norm, we refer for example to [13]. $W_{0}^{1, A}(\Omega)$ is the closure of $\mathcal{D}(\Omega)$ in $W^{1, A}(\Omega)$ with respect to its norm.

Remark 1. (i) We call a solution of problem (1.1) any pair of functions $(u, \chi) \in$ $W^{1, A}(\Omega) \times L^{\infty}(\Omega)$ that satisfies (1.1)ii) in the following weak sense

$$
\int_{\Omega}\left(\frac{a(|\nabla u|)}{|\nabla u|} \nabla u+\chi H(x)\right) \cdot \nabla \zeta d x=0 \quad \forall \zeta \in W_{0}^{1, A}(\Omega) .
$$

(ii) For the existence of a solution of problem (1.1), one usually needs to impose some boundary conditions on $\partial \Omega$, which are typically a mixture of Dirichlet and Neuman conditions. Then under assumptions (1.2) and (1.4), one can prove existence of a solution to problem (1.1) by arguing as in $[16,17,20,21,23]$. The main idea consists in solving an approximating problem using Schauder fixed point theorem, and then passing to the limit using adequate estimates.

Among free boundary problems that fit in the problem (1.1) setting, is the dam problem (see $[4,5,8,12,20,21,22,23]$, which consists in studying the filtration of a fluid through a porous medium $\Omega \subset \mathbb{R}^{n}$, where we look for the fluid pressure or hydrostatic head pressure inside $\Omega$ and the saturated region represented by a function that lies between 0 and 1 . The classical formulation of the dam problem assumes that the flow is governed by Darcy's law i.e.

$$
\mathbf{v}=-a(x)\left(\nabla\left(u+x_{n}\right)\right)=-a(x)(\nabla u+e),
$$


where $u$ is the fluid pressure, $e=(0, \ldots 0,1), \mathbf{v}$ is the fluid velocity and $a(x)$ is the matrix permeability of the porous medium. In this case equation (1.1)ii) reads (see $[17,20]$ )

$$
\operatorname{div}(\mathrm{a}(\mathrm{x})(\nabla \mathrm{u}+\chi \mathrm{e}))=0 \quad \text { in } \mathrm{H}^{-1}(\Omega) .
$$

It is well known that Darcy's law fails to hold for non-Newtonian fluids in which case it is substituted by a power-law of the form $\mathbf{v}=-k|\nabla u|^{p-2} \nabla u$, where $u$ is the fluid hydrostatic head pressure $=$ fluid pressure $+x_{n}, \mathbf{v}$ is the fluid velocity and $k$ is a positive constant. If we set $g=1-\chi$, then equation (1.1)ii) reads (see [4])

$$
\Delta_{p} u-\operatorname{div}(\mathrm{ge})=0 \quad \text { in } \mathrm{W}^{-1, \mathrm{p}^{\prime}}(\Omega) .
$$

In order to take into account the heterogeneity of the medium and the non-Newtonian flow, the following generalization of the above power-law was proposed in [21] (see also $[5,12,22,23]): \mathbf{v}=\mathcal{A}(x, \nabla u)$, where $\mathcal{A}$ is a vector function from $\Omega \times \mathbb{R}^{n}$ to $\mathbb{R}^{n}$ such that $\mathcal{A}(., \xi)$ is measurable, $\mathcal{A}(x,$.$) is continuous$ and monotone, $\mathcal{A}(x, \xi) . \xi \geq \lambda|\xi|^{p}$ and $|\mathcal{A}(x, \xi)| \leq \Lambda|\xi|^{p-1}$ for a.e. $x \in \Omega$ and all $\xi \in \mathbb{R}^{n}$, for some $p>1$ and $\lambda, \Lambda>0$. In this case, (1.1)ii) reads (see $[5,12,21,22,23])$

$$
\operatorname{div}(\mathcal{A}(\mathrm{x}, \nabla \mathrm{u})-\mathrm{g} \mathcal{A}(\mathrm{x}, \mathrm{e}))=0 \quad \text { in } \mathrm{W}^{-1, \mathrm{p}^{\prime}}(\Omega) .
$$

Another application of problem (1.1) arises from the lubrication problem (see $[1,2]$ ) which describes lubrication with cavitation in bearings. The classical formulation of this problem assumes that the flow in a rectangular domain $\Omega$ is governed by Reynolds law i.e. $\operatorname{div}\left(\mathrm{h}^{3}\left(\mathrm{x}_{1}\right) \nabla \mathrm{u}\right)=\mathrm{h}^{\prime}\left(\mathrm{x}_{1}\right)$ in $\{u>0\}$, where $u$ is the fluid pressure, and $h\left(x_{1}\right)$ is the gap between the bearing and the shaft. In this problem there are two unknowns, the fluid pressure $u$ and the fluid relative thickness $0 \leq \chi \leq 1$. If $e_{1}=(1,0)$ then Reynolds law and the incompressibility of the fluid lead to the following version of $(1.1) i i$ ) (see [1,2])

$$
\operatorname{div}\left(\mathrm{h}^{3}\left(\mathrm{x}_{1}\right) \nabla \mathrm{u}-\mathrm{h}\left(\mathrm{x}_{1}\right) \chi \mathrm{e}_{1}\right)=0 \quad \text { in } \mathrm{H}^{-1}(\Omega) .
$$

One more application of problem (1.1) is the thermoelectrical modeling of aluminium electrolysis (see [3]). This model is based on the Fourier law $\mathbf{q}=-k(x) \nabla T$, where $T$ is the aluminium temperature in an electrolytic cell section materialized by a bounded domain $\Omega$ of $\mathbb{R}^{2}$, $\mathbf{q}$ is the heat flux and $k(x)$ is the thermal conductivity. Assuming that $T_{s}$ is the solidification temperature of aluminium, then the problem consists in finding the function $u=T-T_{s} \geq 0$ and a function $0 \leq \chi \leq 1$ that describes the region occupied by the liquid phase $\{u>0\}$. If $h(x)$ represents the heat flux through the free boundary $\partial\{u>0\} \cap \Omega$, then the Fourier law and the conservation of energy equation lead to the following version of (1.1)ii) (see [3])

$$
\operatorname{div}\left(\mathrm{k}(\mathrm{x}) \nabla \mathrm{u}+\mathrm{h}(\mathrm{x}) \chi \mathrm{e}_{1}\right)=0 \quad \text { in } \mathrm{H}^{-1}(\Omega) .
$$

For a more general framework, we refer to $[6,7,9,11,15,24]$. In this paper we generalize results from $[9,11]$ for the p-Laplacian and results in $[6,7]$ in the 
linear case. Regarding the problem with a Newman boundary condition, we refer to [17] for the dam problem, and to [25,27] for a more general framework.

In the first part of the paper, we show interior and boundary Lipschitz continuity. In the second part, under more assumptions on $H$ including $\operatorname{div}(\mathrm{H}) \geq 0$, we establish that the free boundary is represented by a family of lower semicontinuous functions.

Throughout this paper, we shall denote by $B_{r}(x)$ an open ball with center $x$ and radius $r$. If the center is not given, it will be assumed to be the origin.

\section{Interior and boundary Lipschitz continuity}

The first result of this section is the following interior regularity.

Theorem 1. Let $(u, \chi)$ be a solution of (1.1). Then $u \in C_{l o c}^{0,1}(\Omega)$.

We observe that since $H \in L_{l o c}^{\infty}(\Omega)$, we have $u \in C_{l o c}^{0, \alpha}(\Omega)$ for some $\alpha \in(0,1)$ [10]. Consequently the set $\{u>0\}$ is open. Moreover, we have $\Delta_{A} u=-\operatorname{div}(\mathrm{H})$ in $\mathcal{D}^{\prime}(\{u>0\})$ and $\operatorname{div}(\mathrm{H}) \in \mathrm{L}_{\text {loc }}^{\infty}(\Omega)$. So we have $u \in C_{\text {loc }}^{1, \beta}(\{u>0\})$ for some $\beta \in(0,1)[19]$. Therefore to prove Theorem 1 , it is enough to investigate the behavior of $u$ near the free boundary. This is the object of the following lemma.

Lemma 1. Let $x_{0}=\left(x_{01}, \ldots, x_{0 n}\right)$ and $r>0$ such that $B_{r}\left(x_{0}\right) \subset\{u>0\}$, $\bar{B}_{r}\left(x_{0}\right) \subset \Omega$ and $\partial B_{r}\left(x_{0}\right) \cap \partial\{u>0\} \neq \emptyset$. Then there exists a positive constant $C$ depending only on $n, \bar{h}, a_{0}, a^{-1}(\bar{h})$, and $\delta(\Omega)$ (the diameter of $\Omega$ ) such that $\sup _{B_{r / 2}\left(x_{0}\right)} u \leq C r$.

Proof. We start by applying Harnack's inequality (see [19], Corollary 1.4):

$$
\sup _{B_{r / 2}\left(x_{0}\right)} u \leq C\left(\inf _{B_{r / 2}\left(x_{0}\right)} u+r \cdot a^{-1}(\bar{h} \delta(\Omega))\right),
$$

where $C$ is a positive constant depending only on $n, a_{0}$ and $a_{1}$. Therefore, to prove the lemma, it will be enough to establish the inequality

$$
\min _{\bar{B}_{r / 2}\left(x_{0}\right)} u \leq C r
$$

Since $\bar{B}_{r}\left(x_{0}\right) \subset \Omega$, then for $\epsilon \in(0, r)$ small enough, we have $\bar{B}_{r+\epsilon}\left(x_{0}\right) \subset \Omega$, and we can define the following function in the circular ring $D=B_{r+\epsilon}\left(x_{0}\right) \backslash$ $\bar{B}_{r / 2}\left(x_{0}\right): v(x)=k\left(e^{-\alpha \rho^{2}}-e^{-\alpha(r+\epsilon)^{2}}\right)$, where

$$
\begin{aligned}
& \rho=\left|x-x_{0}\right|, \quad k=\frac{m}{e^{-\alpha r^{2} / 4}-e^{-\alpha(r+\epsilon)^{2}}}, \quad m=\min _{\bar{B}_{r / 2}\left(x_{0}\right)} u, \\
& \alpha=\kappa / r^{2}, \quad \kappa=2\left(1+n / a_{0}\right) .
\end{aligned}
$$

We claim that

$$
\Delta_{A} v \geq a(|\nabla v|) / \rho \quad \text { in } D .
$$

Indeed, we first observe that

$$
\Delta_{A} v=\frac{a(|\nabla v|)}{|\nabla v|^{3}}\left\{|\nabla v|^{2} \Delta v+\left(\frac{a^{\prime}(|\nabla v|)}{a(|\nabla v|)}|\nabla v|-1\right) \sum_{i, j} \frac{\partial v}{\partial x_{i}} \frac{\partial v}{\partial x_{j}} \frac{\partial^{2} v}{\partial x_{i} \partial x_{j}}\right\} .
$$


Moreover, we have

$$
\begin{aligned}
& \nabla v=-2 \alpha k e^{-\alpha \rho^{2}}\left(x-x_{0}\right), \quad|\nabla v|=2 \alpha k \rho e^{-\alpha \rho^{2}}, \\
& \Delta v=-2 \alpha k e^{-\alpha \rho^{2}}\left(n-2 \alpha \rho^{2}\right), \\
& \frac{\partial^{2} v}{\partial x_{i} \partial x_{j}}=-2 \alpha k e^{-\alpha \rho^{2}}\left(\delta_{i j}-2 \alpha\left(x_{i}-x_{0 i}\right)\left(x_{j}-x_{0 j}\right)\right), \\
& \sum_{i, j} \frac{\partial v}{\partial x_{i}} \frac{\partial v}{\partial x_{j}} \frac{\partial^{2} v}{\partial x_{i} \partial x_{j}}=-(2 \alpha k)^{3} \rho^{2} e^{-3 \alpha \rho^{2}}\left(1-2 \alpha \rho^{2}\right) .
\end{aligned}
$$

Taking into account the fact that

$$
1-2 \alpha \rho^{2}=1-2 \frac{\kappa}{r^{2}} \rho^{2} \leq 1-2 \frac{\kappa}{r^{2}}\left(\frac{r}{2}\right)^{2}=1-\frac{\kappa}{2}<0,
$$

we get by substituting the above formulas in (2.2)

$$
\begin{aligned}
\Delta_{A} v & =-(2 \alpha k)^{3} \rho^{2} e^{-3 \alpha \rho^{2}} \frac{a(|\nabla v|)}{|\nabla v|^{3}}\left\{n-1+\frac{a^{\prime}(|\nabla v|)}{a(|\nabla v|)}|\nabla v|\left(1-2 \alpha \rho^{2}\right)\right\} \\
& \geq-(2 \alpha k)^{3} \rho^{2} e^{-3 \alpha \rho^{2}} \frac{a(|\nabla v|)}{|\nabla v|^{3}}\left\{n-1+a_{0}\left(1-\frac{\kappa}{2}\right)\right\} \quad \text { by }(1.2) \\
& =-\frac{a(|\nabla v|)}{\rho}\left(n-1+a_{0}\left(1-\frac{\kappa}{2}\right)\right)=\frac{a(|\nabla v|)}{\rho} .
\end{aligned}
$$

Hence (2.1) holds, which leads by using (1.5) to

$$
\begin{aligned}
\Delta_{A} v & +\operatorname{div}(\mathrm{H}) \geq \mathrm{a}(|\nabla \mathrm{v}|) / \rho-\overline{\mathrm{h}} \\
& \geq \frac{1}{(r+\epsilon)} a\left(2 \frac{\kappa}{r^{2}} \cdot \frac{m e^{-\frac{\kappa}{r^{2}}(r+\epsilon)^{2}}}{e^{-\frac{\kappa}{4}}-e^{-\frac{\kappa}{r^{2}}(r+\epsilon)^{2}}} \cdot \frac{r}{2}\right)-\bar{h}=\theta(r) .
\end{aligned}
$$

- If $\theta(r) \leq 0$, then $a\left(\frac{\kappa}{r} \cdot \frac{m e^{-\frac{\kappa}{r^{2}}(r+\epsilon)^{2}}}{e^{-\frac{\kappa}{4}}-e^{-\frac{\kappa}{r^{2}}(r+\epsilon)^{2}}}\right) \leq \bar{h}(r+\epsilon)$, and by letting $\epsilon \rightarrow 0$, we get $a\left(\frac{\kappa}{r} \cdot \frac{m e^{-\kappa}}{e^{-\frac{\kappa}{4}}-e^{-\kappa}}\right) \leq \bar{h} r \leq \bar{h} \delta(\Omega)$, which leads to

$$
m \leq a^{-1}(\bar{h} \delta(\Omega)) \frac{\left(e^{\frac{3}{4} \kappa}-1\right)}{\kappa} r=C(n, a, \bar{h}, \delta(\Omega)) r
$$

and the lemma follows.

- If $\theta(r)>0$, we deduce from $(2.3)$, since $(v-u)^{+} \in W_{0}^{1, A}(D)$ and $(v-u)^{+} \geq 0$, that

$$
\int_{D}\left(\frac{a(|\nabla v|)}{|\nabla v|} \nabla v+H(x)\right) \cdot \nabla(v-u)^{+} \leq 0 .
$$

From $(1.1)(i i)$, we also have

$$
\int_{D}\left(\frac{a(|\nabla u|)}{|\nabla u|} \nabla u+\chi H(x)\right) \cdot \nabla(v-u)^{+}=0 .
$$


Subtracting (2.5) from (2.4), we get

$$
\int_{D}\left(\frac{a(|\nabla v|)}{|\nabla v|} \nabla v-\frac{a(|\nabla u|)}{|\nabla u|} \nabla u\right) \cdot \nabla(v-u)^{+} d x \leq \int_{D}(\chi-1) H(x) \cdot \nabla(v-u)^{+} d x
$$

which can be written by using (1.4) and the fact that $\chi=1$ a.e. in $\{u>0\}$

$$
\begin{aligned}
& \int_{D \cap\{u>0\}}\left(\frac{a(|\nabla v|)}{|\nabla v|} \nabla v-\frac{a(|\nabla u|)}{|\nabla u|} \nabla u\right) \cdot \nabla(v-u)^{+} d x \\
& \quad \leq \int_{D \cap\{u=0\}}(\chi-1) H(x) \cdot \nabla v d x-\int_{D \cap\{u=0\}} \frac{a(|\nabla v|)}{|\nabla v|}|\nabla v|^{2} d x \\
& \quad \leq \int_{D \cap\{u=0\}}|\nabla v|(\bar{h}-a(|\nabla v|)) d x .
\end{aligned}
$$

If $\int_{D \cap\{u=0\}}|\nabla v|(\bar{h}-|a(|\nabla v|)|) d x \leq 0$, then we would get by taking into account (1.3) that $\nabla(v-u)^{+}=0$ in $B_{r}\left(x_{0}\right)$. Since $(v-u)^{+}=0$ on $\partial B_{r}\left(x_{0}\right)$, this leads to $v \leq u$ in $\overline{D \cap B_{r}\left(x_{0}\right)}$.

Given that $v>0$ on $\partial B_{r}\left(x_{0}\right)$ and $\partial B_{r}\left(x_{0}\right) \cap \partial\{u>0\} \neq \emptyset$, we would get a contradiction. Hence

$$
\int_{D \cap\{u=0\}}|\nabla v|(\bar{h}-a(|\nabla v|)) d x>0 .
$$

Since $|\nabla v|=2 k \alpha \rho e^{-\alpha \rho^{2}}$ and $\kappa>2$, we have

$$
\frac{d}{d \rho}|\nabla v|=2 k \alpha e^{-\alpha \rho^{2}}\left(1-2 \kappa \frac{\rho^{2}}{r^{2}}\right) \leq 2 k \alpha e^{-\alpha \rho^{2}}(1-\kappa / 2)<0 .
$$

Therefore $|\nabla v|$ is non-increasing with respect to $\rho$. It follows then from (2.6) that $a(|\nabla v|)_{\left.\right|_{\partial B_{r+\epsilon}\left(x_{0}\right)}}=a\left(2 k \alpha(r+\epsilon) e^{-\alpha(r+\epsilon)^{2}}\right)<\bar{h}$ i.e.

$$
\frac{2 m \kappa(r+\epsilon) e^{-\alpha(r+\epsilon)^{2}}}{r^{2}\left(e^{-\alpha r^{2} / 4}-e^{-\alpha(r+\epsilon)^{2}}\right)}<a^{-1}(\bar{h})
$$

Letting $\epsilon \rightarrow 0$, we obtain $\frac{2 m \kappa e^{-\kappa}}{r\left(e^{-\kappa / 4}-e^{-\kappa}\right)} \leq a^{-1}(\bar{h})$, which leads to

$$
m \leq \frac{a^{-1}(\bar{h})}{2 \kappa}\left(e^{3 \kappa / 4}-1\right) r=C(\bar{h}, \kappa, a) r
$$

Proof. (of Theorem 1) The proof is based on Lemma 1 and arguments similar to those in the p-Laplacian case [11]. In particular, we use the scaling function

$$
v(y)=u\left(x_{0}+R y\right) / R \quad \text { for } \quad y \in B_{1},
$$


which satisfies the equation

$$
\Delta_{A} v=-R(\operatorname{div} H)\left(x_{0}+R y\right) \quad \text { in } \quad B_{1} \quad \text { if } \quad B_{R}\left(x_{0}\right) \subset\{u>0\} .
$$

Then by applying the estimate from [19], Theorem 1.7, we get for some positive constant $C(n, a, M, R)$ that $\sup _{B_{1 / 2}}|\nabla v| \leq C(n, a, M, R)$.

Now we assume that $u=0$ on a nonempty subset $T$ of $\partial \Omega$, and we study the Lipschitz continuity of $u$ up to $T$. To this end we assume the uniform exterior sphere condition satisfied locally on $T$ i.e. for each open and connected subset $S \subset \subset T$

$$
\exists R>0 \text { such that } \forall y \in S \quad \exists z \in \mathbb{R}^{n} \backslash \bar{\Omega} \quad \bar{B}_{R}(z) \cap S=\{y\} .
$$

Without loss of generality, we can assume that $R<\frac{1}{3} \operatorname{dist}(S, \partial \Omega \backslash T)>0$, where dist is the distance between two sets. Then we state our second result.

Theorem 2. For any solution $(u, \chi)$ of $(1.1)$, we have $u \in C_{l o c}^{0,1}(\Omega \cup T)$.

The proof of Theorem 2 is based on Lemma 2. The rest of the proof will be omitted, since it can be easily obtained using arguments similar to those in the proof of Theorem 1 [11] and taking into account the above remark at the end of the proof of Theorem 1.

Lemma 2. Let $S$ be an open connected subset of $T$ such that $S \subset \subset T$. Then there exists a positive constant $C$ depending only on $n, a, M, \bar{h}, \delta(\Omega)$ and $R$ such that

$$
u(x) \leq C|x-y| \quad \forall x \in \Omega \quad \forall y \in S .
$$

Proof. Let $y \in S, z=y+R \nu$, where $\nu$ is the outward unit normal vector to $\partial \Omega$ at $y$ such that $B_{R}(z) \cap \partial \Omega=\{y\}$. Then we consider the function $v(x)=\vartheta(d(x))$, where $d$ and $\vartheta$ are given by $d(x)=|x-z|-R$,

$$
\vartheta(t)=\int_{0}^{t} a^{-1}\left(\left(a\left(\frac{M}{R}\right)+\frac{\bar{h} R}{n-1}\right) e^{\frac{n-1}{R}(D-s)}-\frac{\bar{h} R}{n-1}\right) d s \text { and } D=\delta(\Omega) .
$$

Then it is easy to verify that the following properties of $\vartheta$ hold:

$$
\begin{aligned}
& \vartheta(0)=0, \vartheta(R) \geq M \text { and for all } t \in[0, D], \\
& \vartheta^{\prime}(t)=a^{-1}\left(\left(a\left(\frac{M}{R}\right)+\frac{\bar{h} R}{n-1}\right) e^{\frac{n-1}{R}(D-t)}-\frac{\bar{h} R}{n-1}\right)>0, \\
& \vartheta^{\prime}(D)=\frac{M}{R} \leq \vartheta^{\prime}(t) \leq \vartheta^{\prime}(0)=a^{-1}\left(\left(a\left(\frac{M}{R}\right)+\frac{\bar{h} R}{n-1}\right) e^{\frac{n-1}{R} D}-\frac{\bar{h} R}{n-1}\right), \\
& a\left(\vartheta^{\prime}(t)\right) \vartheta^{\prime \prime}(t)+\frac{n-1}{R} a\left(\vartheta^{\prime}(t)\right)+\bar{h}=0 .
\end{aligned}
$$

We also have

$$
\frac{\partial v}{\partial x_{i}}=\vartheta^{\prime}(d(x)) \frac{\partial d}{\partial x_{i}}=\vartheta^{\prime}(d(x)) \frac{x_{i}-z_{i}}{|x-z|}
$$




$$
\begin{aligned}
& \frac{a(|\nabla v|)}{|\nabla v|} \nabla v=a\left(\vartheta^{\prime}(d(x))\right) \nabla d(x), \quad \frac{\partial^{2} d}{\partial x_{i}^{2}}=\frac{1}{|x-z|}-\frac{\left(x_{i}-z_{i}\right)^{2}}{|x-z|^{3}} \\
& \frac{\partial}{\partial x_{i}}\left(\frac{a(|\nabla v|)}{|\nabla v|} \frac{\partial v}{\partial x_{i}}\right)=a^{\prime}\left(\vartheta^{\prime}(d(x))\right) \vartheta^{\prime \prime}(d(x))\left(\frac{\partial d}{\partial x_{i}}\right)^{2}+a\left(\vartheta^{\prime}(d(x))\right) \frac{\partial^{2} d}{\partial x_{i}^{2}} \\
& \Delta_{A} v=a^{\prime}\left(\vartheta^{\prime}(d(x))\right) \vartheta^{\prime \prime}(d(x))+\frac{n-1}{|x-z|} a\left(\vartheta^{\prime}(d(x))\right)
\end{aligned}
$$

Therefore, since $|x-z|>R$ for all $x$ in $\Omega$, we obtain

$$
\Delta_{A} v+\operatorname{div}(H) \leq 0 \quad \text { in } \quad \Omega .
$$

Next, we claim that

$$
u(x) \leq v(x) \quad \text { for all } \quad x \in \partial \Omega .
$$

Indeed, for $x \in T$, we have $u(x)=0 \leq v(x)$. For $x \in \partial \Omega \backslash T$, we have $|x-y| \leq|x-z|+|z-y|=|x-z|+R$, which leads to $|x-z| \geq|x-y|-R \geq$ $\operatorname{dist}(S, \partial \Omega \backslash T)-R>3 R-R=2 R$. Hence we get $v(x) \geq \vartheta(R) \geq M \geq u(x)$ on $\partial \Omega \backslash T$.

Now thanks to $(2.8)$, we have $(u-v)^{+} \in W_{0}^{1, A}(\Omega)$. Using this function in (1.1) $\mathrm{ii}$ ) and in (2.7), we obtain

$$
\begin{aligned}
& \int_{\Omega} \frac{a(|\nabla u|)}{|\nabla u|} \nabla u \cdot \nabla(u-v)^{+}=-\int_{\Omega} \chi H(x) \cdot \nabla(u-v)^{+} d x, \\
& -\int_{\Omega} \frac{a(|\nabla v|)}{|\nabla v|} \nabla v \cdot \nabla(u-v)^{+} d x \leq \int_{\Omega} H(x) \cdot \nabla(u-v)^{+} d x .
\end{aligned}
$$

Taking into account that $\chi=1$ a.e. in $\{u>0\}$ and adding (2.9) and (2.10), we obtain

$$
\int_{\Omega}\left(\frac{a(|\nabla u|)}{|\nabla u|} \nabla u-\frac{a(|\nabla v|)}{|\nabla v|} \nabla v\right) \cdot \nabla(u-v)^{+} d x \leq 0,
$$

which leads by $(1.3)$ to $\nabla(u-v)^{+}=0$ a.e. in $\Omega$, and therefore $(u-v)^{+}$is constant in $\Omega$. Since $u \leq v$ on $\partial \Omega$, we get $u \leq v$ in $\Omega$. We conclude that for all $x \in \Omega$ and $y \in S$, we have

$$
\begin{aligned}
& u(x) \leq v(x)=|v(x)-v(y)| \leq \sup _{x \in \Omega}|\nabla v(x)||x-y| \leq\left(\sup _{t \in[0, D]} \vartheta^{\prime}(t)\right)|x-y| \\
& =\vartheta^{\prime}(0)|x-y|=C(n, a, M, \bar{h}, D, R)|x-y| .
\end{aligned}
$$

\section{The free boundary}

In this section, we assume that the vector function $H$ satisfies the following assumptions for some positive constants $\underline{h}$ and $\bar{h}$ :

$$
\begin{aligned}
& 0<\underline{h} \leq H_{n} \leq \bar{h} \quad \text { a.e. in } \Omega, \quad H \in C^{0,1}(\bar{\Omega}), \\
& \operatorname{div}(\mathrm{H}) \geq 0 \quad \text { a.e. in } \Omega .
\end{aligned}
$$


By using $\min (u / \epsilon, 1) \zeta$ with $\zeta \in \mathcal{D}(\Omega), \zeta \geq 0$ as a test function for (1.1)ii) and arguing as in [7], one can establish the following important inequality:

$$
\operatorname{div}(\chi \mathrm{H})-\chi(\{\mathrm{u}>0\}) \operatorname{div}(\mathrm{H}) \leq 0 \quad \text { in } \quad \mathcal{D}^{\prime}(\Omega) .
$$

As a consequence of (3.3), we will derive a weak monotonicity of the function $\chi$, that will be used to express the free boundary as a union of graphs of a family of functions. More precisely, we consider the following differential equation

$$
(E(\omega, h))\left\{\begin{aligned}
X^{\prime}(t, \omega, h) & =H(X(t, \omega, h)), \\
X(0, \omega, h) & =(\omega, h),
\end{aligned}\right.
$$

where $h \in \pi_{x_{n}}(\Omega)$ and $\omega \in \pi_{x^{\prime}}\left(\Omega \cap\left\{x_{n}=h\right\}\right), x^{\prime}=\left(x_{1}, \ldots, x_{n-1}\right), \pi_{x^{\prime}}$ and $\pi_{x_{n}}$ are respectively the orthogonal projections on the hyperplane $\left\{x_{n}=0\right\}$ and the $x_{n}$-axis. Then we denote by $X(., \omega, h)$ the maximal solution of $E(\omega, h)$ defined on the interval $\left(\alpha_{-}(\omega, h), \alpha_{+}(\omega, h)\right)$. We deduce from (1.4) that we have

$$
\left|X\left(t_{1}, \omega, h\right)-X\left(t_{2}, \omega, h\right)\right| \leq \bar{h}\left|t_{1}-t_{2}\right| \quad \forall t_{1}, t_{2} \in\left(\alpha_{-}(\omega, h), \alpha_{+}(\omega, h)\right) .
$$

It follows that the limits $\lim _{t \rightarrow \alpha_{-}(\omega, h)^{+}} X(t, \omega, h)$ and $\lim _{t \rightarrow \alpha_{+}(\omega, h)^{-}} X(t, \omega, h)$ both exit, which we shall denote respectively by $X\left(\alpha_{-}(\omega, h), \omega, h\right)$ and $X\left(\alpha_{+}(\omega, h), \omega, h\right)$, and observe that we have necessarily $X\left(\alpha_{-}(\omega, h), \omega, h\right) \in \partial \Omega \cap\left\{x_{n}<h\right\}$ and $X\left(\alpha_{+}(\omega, h), \omega, h\right) \in \partial \Omega \cap\left\{x_{n}>h\right\}$.

For simplicity, we will drop the dependence on $h$ in the sequel. Now, we recall for the reader's convenience the following technical properties and definitions established in [7]:

- $\alpha_{+}$and $\alpha_{-}$are uniformly bounded.

- For each $h \in \pi_{x_{n}}(\Omega)$, we define the set

$$
D_{h}=\left\{(t, \omega) / \omega \in \pi_{x^{\prime}}\left(\Omega \cap\left\{x_{n}=h\right\}\right), t \in\left(\alpha_{-}(\omega), \alpha_{+}(\omega)\right)\right\}
$$

and consider the mapping

$$
\begin{aligned}
T_{h}: D_{h} & \longrightarrow T_{h}\left(D_{h}\right), \\
(t, \omega) & \longmapsto T_{h}(t, \omega)=\left(T_{h}^{1}, \ldots, T_{h}^{n}\right)(t, \omega)=X(t, \omega) .
\end{aligned}
$$

- $\Omega=\bigsqcup_{h \in \pi_{x_{n}}(\Omega)} T_{h}\left(D_{h}\right), \quad T_{h}$ is one to one, $\quad T_{h}$ and $T_{h}^{-1}$ are $C^{0,1}$.

- The determinant $Y_{h}(t, \omega)$ of the Jacobian matrix of the mapping $T_{h}$, satisfies:

i) $Y_{h}(t, \omega)=-H_{n}(\omega, h) \exp \left(\int_{0}^{t}(\operatorname{div} H)(X(s, \omega)) d s\right)$ a.e. in $D_{h}$.

ii) $\underline{h} \leq-Y_{h}(t, \omega) \leq C \bar{h}, \quad C>0, \quad$ a.e. in $D_{h}$.

Using (3.3) and arguing as in the proof of Theorem 1 of [7], we can establish the following monotonicity of $\chi$

$$
\frac{\partial}{\partial t}\left(\chi o T_{h}\right) \leq 0 \quad \text { in } \mathcal{D}^{\prime}\left(D_{h}\right)
$$


Property (3.4) means that $\chi$ decreases along the orbits of the differential equation $(E(w, h))$. The consequence on $u$ is materialized in the next key theorem which is the main idea in the parametrization of the free boundary.

Theorem 3. Let $(u, \chi)$ be a solution of (1.1) and $x_{0}=T_{h}\left(t_{0}, \omega_{0}\right) \in T_{h}\left(D_{h}\right)$.

i) If $u o T_{h}\left(t_{0}, \omega_{0}\right)>0$, then there exists $\epsilon>0$ such that

$$
u o T_{h}(t, \omega)>0 \quad \forall(t, \omega) \in C_{\epsilon}=\left\{(t, \omega) \in D_{h} /\left|\omega-\omega_{0}\right|<\epsilon, t<t_{0}+\epsilon\right\} .
$$

ii) If $u o T_{h}\left(t_{0}, \omega_{0}\right)=0$, then $u o T_{h}\left(t, \omega_{0}\right)=0 \quad \forall t \geq t_{0}$.

To prove Theorem 3, we need the following strong maximum principle.

Lemma 3. Let $u \in W^{1, A}(U) \cap C^{1}(U) \cap C^{0}(\bar{U})$ such that $u \geq 0$ in $U$ and $\Delta_{A} u \leq 0$ in $U$. Then $u \equiv 0$ in $U$ or $u>0$ in $U$.

The proof of Lemma 3 follows from the next Lemma 4 as in [18] p. 333.

Lemma 4. Let $u \in W^{1, A}(U) \cap C^{1}(\bar{U})$ such that $\Delta_{A} u \leq 0$ in $U$ and $u\left(x_{0}\right)<$ $u(x)$ for all $x \in U$, where $x_{0} \in \partial B_{R}\left(x_{1}\right)$ and $B_{R}\left(x_{1}\right) \subset U$. Then the outer normal derivative of $u$ at $x_{0}$, satisfies $\frac{\partial u}{\partial \nu}\left(x_{0}\right)<0$.

Proof. We consider the standard function $v$ defined by

$$
v(x)=e^{-\alpha r^{2}}-e^{-\alpha R^{2}} \text { for } x \in D=B_{R}\left(x_{1}\right) \backslash \bar{B}_{R / 2}\left(x_{1}\right),
$$

where $r=\left|x-x_{1}\right| \in(R / 2, R), \alpha=\kappa /(R / 2)^{2}=4 \kappa / R^{2}$, and $\kappa$ is a positive parameter such that $\frac{1}{2}<\kappa<2\left(1+\frac{n-2}{a_{0}}\right)$.

Let $\epsilon=\min _{\partial B_{R / 2}\left(x_{1}\right)}\left(u-u\left(x_{0}\right)\right) / \max _{\partial B_{R / 2}\left(x_{1}\right)} v>0$, and observe that

$$
\epsilon v \leq u-u\left(x_{0}\right) \quad \text { on } \partial D
$$

To establish the Lemma, we will compare $u-u\left(x_{0}\right)$ with respect to $\epsilon v$. We claim that

$$
\Delta_{A}(\epsilon v) \geq a(\epsilon|\nabla v|) / r \geq 0 \quad \text { in } D .
$$

Indeed, we first observe from (2.2) that

$$
\Delta_{A}(\epsilon v)=\frac{a(\epsilon|\nabla v|)}{|\nabla v|^{3}}\left\{|\nabla v|^{2} \Delta v+\left(\frac{a^{\prime}(\epsilon|\nabla v|)}{a(\epsilon|\nabla v|)}|\epsilon \nabla v|-1\right) \sum_{i, j} \frac{\partial v}{\partial x_{i}} \frac{\partial v}{\partial x_{j}} \frac{\partial^{2} v}{\partial x_{i} \partial x_{j}}\right\} .
$$

Moreover, we have

$$
\begin{aligned}
& \nabla v=-2 \alpha k e^{-\alpha r^{2}}\left(x-x_{1}\right),|\nabla v|=2 \alpha r e^{-\alpha r^{2}}, \Delta v=-2 \alpha e^{-\alpha r^{2}}\left(n-2 \alpha r^{2}\right), \\
& \frac{\partial^{2} v}{\partial x_{i} \partial x_{j}}=-2 \alpha e^{-\alpha r^{2}}\left(\delta_{i j}-2 \alpha\left(x_{i}-x_{1 i}\right)\left(x_{j}-x_{1 j}\right)\right) \\
& \sum_{i, j} \frac{\partial v}{\partial x_{i}} \frac{\partial v}{\partial x_{j}} \frac{\partial^{2} v}{\partial x_{i} \partial x_{j}}=-(2 \alpha)^{3} r^{2} e^{-3 \alpha r^{2}}\left(1-2 \alpha r^{2}\right) .
\end{aligned}
$$


Taking into account the fact that

$$
1-2 \alpha r^{2}=1-2 \frac{\kappa}{r^{2}} r^{2} \leq 1-2 \frac{\kappa}{(R / 2)^{2}}\left(\frac{R}{2}\right)^{2}=1-2 \kappa<0,
$$

we get by substituting the above formulas in (3.7)

$$
\begin{gathered}
\Delta_{A}(\epsilon v)=(2 \alpha)^{3} r^{2} e^{-3 \alpha r^{2}} \frac{a(\epsilon|\nabla v|)}{|\nabla v|^{3}}\left\{n-1+\frac{a^{\prime}(\epsilon|\nabla v|)}{a(\epsilon|\nabla v|)}|\epsilon \nabla v|\left(1-2 \alpha r^{2}\right)\right\} \\
\geq(2 \alpha)^{3} r^{2} e^{-3 \alpha r^{2}} \frac{a(\epsilon|\nabla v|)}{|\nabla v|^{3}}\left\{n-1+a_{0}\left(1-\frac{\kappa}{2}\right)\right\} \quad \text { by (1.2) } \\
=\frac{a(|\nabla v|)}{r}\left(n-1+a_{0}\left(1-\frac{\kappa}{2}\right)\right) \geq \frac{a(\epsilon|\nabla v|)}{r} .
\end{gathered}
$$

Hence (3.6) holds. Using (3.6) and the fact that $\Delta_{A} u \leq 0$, we obtain

$$
\Delta_{A}(\epsilon v) \geq 0 \geq \Delta_{A}\left(u-u\left(x_{0}\right)\right) \quad \text { in } D .
$$

Now taking into account (3.5) and (3.8), and using the weak maximum principle for the $A$-Laplacian, we get

$$
\epsilon v \leq u-u\left(x_{0}\right) \quad \text { in } D .
$$

To conclude, let $\nu$ be the exterior unit normal vector to $\partial B_{R}\left(x_{1}\right)$ at $x_{0}$. We infer from (3.9) for $t$ positive and small enough so that $x_{0}-t \nu \in D$

$$
\frac{u\left(x_{0}-t \nu\right)-u\left(x_{0}\right)}{t} \geq \epsilon \frac{v\left(x_{0}-t \nu\right)-v\left(x_{0}\right)}{t} .
$$

Letting $t \longrightarrow 0$, we obtain

$$
\begin{aligned}
-\frac{\partial u}{\partial \nu}\left(x_{0}\right) & \geq \epsilon \cdot\left(-\frac{\partial v}{\partial \nu}\left(x_{0}\right)\right), \\
\frac{\partial u}{\partial \nu}\left(x_{0}\right) & \leq \epsilon \frac{\partial v}{\partial \nu}\left(x_{0}\right)=\epsilon \cdot\left(-2 \alpha R e^{-\alpha R^{2}}\right)<0 .
\end{aligned}
$$

Proof. (of Theorem 3) It is enough to verify $i$ ). By continuity, there exists $\epsilon>0$ such that $u o T_{h}(t, \omega)>0 \forall(t, \omega) \in\left(t_{0}-\epsilon, t_{0}+\epsilon\right) \times B_{\epsilon}\left(\omega_{0}\right)=Q_{\epsilon}$.

By $(1.1) i)$, we have $\chi o T_{h}(t, \omega)=1$ for a.e. $(t, \omega) \in Q_{\epsilon}$. Using (3.4) and the fact that $\chi o T_{h} \leq 1$, we get $\chi o T_{h}=1$ a.e. in $C_{\epsilon}$, i.e. $\chi=1$ a.e. in $T_{h}\left(C_{\epsilon}\right)$.

From (1.1)ii) and (3.2), we get $\triangle_{A} u=-\operatorname{div}(H) \leq 0$ in $\mathcal{D}^{\prime}\left(T_{h}\left(C_{\epsilon}\right)\right)$. Given that $u \geq 0$ in $\Omega$ and $u>0$ in $T_{h}\left(Q_{\epsilon}\right) \subset T_{h}\left(C_{\epsilon}\right)$, we conclude by Lemma 3 , that $u>0$ in $T_{h}\left(C_{\epsilon}\right)$.

Remark 2. Thanks to Theorem 3, we can define for each $h \in \pi_{x_{n}}(\Omega)$, the following function on $\pi_{x^{\prime}}\left(\Omega \cap\left\{x_{n}=h\right\}\right)$ :

$$
\phi_{h}(\omega)=\left\{\begin{array}{cc}
\sup \{t: & \left.(t, \omega) \in D_{h}, \quad u o T_{h}(t, \omega)>0\right\} \\
& \text { if this set is not empty } \\
\alpha_{-}(\omega), & \text { otherwise. }
\end{array}\right.
$$


Then one can easily check as in [5] that $\phi_{h}$ is lower semi-continuous at each $\omega \in \pi_{x^{\prime}}\left(\Omega \cap\left\{x_{n}=h\right\}\right)$ such that $T_{h}\left(\phi_{h}(\omega), \omega\right) \in \Omega$ and that $\left\{u o T_{h}(t, \omega)>0\right\} \cap$ $D_{h}=\left\{t<\phi_{h}(\omega)\right\}$.

\section{Acknowledgements}

The authors are grateful for the facilities and excellent research conditions provided by Fields Institute where part of this research work was carried out.

\section{References}

[1] S.J. Alvarez and J. Carrillo. A free boundary problem in theory of lubrication. Communications in Partial Differential Equations, 19(11-12):1743-1761, 1994. https://doi.org/10.1080/03605309408821072.

[2] G. Bayada and M. Chambat. Nonlinear variational formulation for a cavitation problem in lubrication. Journal of Mathematical Analysis and Applications, 90(2):286-298, 1982. https://doi.org/10.1016/0022-247X(82)90061-0.

[3] A. Bermúdez, M.C. Muñiz and P. Quintela. Existence and uniqueness for a free boundary problem in aluminum electrolysis. Journal of Mathematical Analysis and Applications, 191(3):497-527, 1995. https://doi.org/10.1006/jmaa.1995.1145.

[4] J. Carrillo and A. Lyaghfouri. On the dam problem with nonlinear Darcy's laws and Dirichlet boundary conditions. Annali della Scuola Normale Superiore di Pisa Cl. Sci., 26(4):453-505, 1998.

[5] S. Challal and A. Lyaghfouri. A filtration problem through a heterogeneous porous medium. Interfaces and Free Boundaries, 6(1):55-79, 2004. https://doi.org/10.4171/IFB/91.

[6] S. Challal and A. Lyaghfouri. On the continuity of the free boundary in problems of type $\operatorname{div}(a(x) \nabla u)=-(\chi(u) h(x))_{x_{1}}$. Nonlinear Analysis : Theory, Methods and Applications, 62(2):283-300, 2005. https://doi.org/10.1016/j.na.2005.02.115.

[7] S. Challal and A. Lyaghfouri. On a class of free boundary problems of type $\operatorname{div}(a(X) \nabla u)=-\operatorname{div}(\chi(u) H(X))$. Differential and Integral Equations, 19(5):481-516, 2006.

[8] S. Challal and A. Lyaghfouri. On the dam problem with two fluids governed by a nonlinear Darcy's law. Advances in Differential Equations, 11(8):841-892, 2006.

[9] S. Challal and A. Lyaghfouri. On the continuity of the free boundary in the problem $\Delta_{p} u=-(h(x, y) \chi(u))_{x}$. Applicable Analysis, 86(9):1177-1184, 2007. https://doi.org/10.1080/00036810701620601.

[10] S. Challal and A. Lyaghfouri. Hölder continuity of solutions to the A-Laplace equation involving measures. Communications in Pure and Applied Analysis, 8(5):1577-1583, 2009. https://doi.org/10.3934/cpaa.2009.8.1577.

[11] S. Challal and A. Lyaghfouri. Lipschitz continuity of solutions of a free boundary problem involving the p-Laplacian. Journal of Mathematical Analysis and Applications, 355(2):700-707, 2009. https://doi.org/10.1016/j.jmaa.2009.02.012. 
[12] S. Challal and A. Lyaghfouri. The heterogeneous dam problem with leaky boundary condition. Communications in Pure and Applied Analysis, 10(1):93-125, 2011. https://doi.org/10.3934/cpaa.2011.10.93.

[13] S. Challal, A. Lyaghfouri and J.F. Rodrigues. On the A-Obstacle problem and the Hausdorff measure of its free boundary. Annali di Matematica Pura ed Applicata, 191(1):113-165, 2012. https://doi.org/10.1007/s10231-010-0177-7.

[14] S. Challal, A. Lyaghfouri, J.F. Rodrigues and R. Teymurazyan. On the regularity of the free boundary for a class of quasilinear obstacle problems. Interfaces and Free Boundaries, 16(3):359-394, 2014. https://doi.org/10.4171/IFB/323.

[15] M. Chipot. On the continuity of the free boundary in some class of twodimensional problems. Interfaces and Free Boundaries, 3(1):81-99, 2001. https://doi.org/10.4171/IFB/33.

[16] M. Chipot and A. Lyaghfouri. The dam problem with nonlinear Darcy's law and leaky boundary conditions. Mathematical Methods in the Applied Sciences, 20(12):1045-1068, 1997.

[17] M. Chipot and A. Lyaghfouri. The dam problem for linear Darcy's law and nonlinear leaky boundary conditions. Advances in Differential Equations, 3(1):150, 1998.

[18] L.C. Evans. Partial Differential Equations. Graduate Studies in Mathematics, AMS, 2010.

[19] G.M. Lieberman. The natural generalization of the natural conditions of Ladyzhenskaya and Uraltseva for elliptic equations. Communications in Partial Differential Equations, 16(2-3):311-361, 1991. https://doi.org/10.1080/03605309108820761.

[20] A. Lyaghfouri. The inhomogeneous dam problem with linear Darcy's law and Dirichlet boundary conditions. Mathematical Models and Methods in Applied Sciences, 8(6):1051-1077, 1996. https://doi.org/10.1142/S0218202596000432.

[21] A. Lyaghfouri. A unified formulation for the dam problem. Rivista di Matematica della Università di Parma, 6(1):113-148, 1998.

[22] A. Lyaghfouri. A free boundary problem for a fluid flow in a heterogeneous porous medium. Annali dell'Universita di Ferrara, 49(1):209-262, 2003.

[23] A. Lyaghfouri. The dam Problem. Handbook of Differential Equations, Stationary Partial Differential Equations, volume 3. North Holland, 2006.

[24] A. Lyaghfouri. On the Lipschitz continuity of the solutions of a class of elliptic free boundary problems. Journal of Applied Analysis, 14(2):165-181, 2008.

[25] A. Lyaghfouri and A. Saadi. Free boundary problems with Neuman boundary condition. Electronic Journal of Differential Equations, 2019(114):1-13, 2019.

[26] J.F. Rodrigues and R. Teymurazyan. On the two obstacles problem in OrliczSobolev spaces and applications. Complex Variables and Elliptic Equations, 56(7-9):769-787, 2011. https://doi.org/10.1080/17476933.2010.505016.

[27] A. Saadi. Continuity of the free boundary in elliptic problems with Neuman boundary condition. Electronic Journal of Differential Equations, 2015(160):116, 2015. 